W. S. LETOCHOW

Laserspektroskopie

DIERCK-EKKEHARD LIEBSCHER

Relativitätstheorie

mit Zirkel und Lineal

Wolfgang MeLING

Digitalrechner

in der elektronischen Meßtechnik

Teil 1: Meßmethodik

Teil 2: Gerätetechnik

und Anwendungen

\section{I. MiroschNITSCHENKO}

Kosmische Strahlung

im interplanetaren Raum

PETER PAUfler

GUSTAV E. R. SCHULZE

Physikalische Grundlagen

mechanischer Festkörpereigenschaften

Teil I und II

ULRICH RÜSEBERG

Quantenmechanik und Philosophie

ALBRecht ROST

Messung

dielektrischer Stoffeigenschaften

J. V. SAČKOV

Wahrscheinlichkeit und Struktur

E. M. SAWIZKI

Perspektiven der Metallforschung

WOLFGANG SCHÄFER

Theoretische Grundlagen

der Stabilität technischer Systeme

ERNST SCHMUTZER

Symmetrien und Erhaltungssätze

der Physik

VOLKMAK SCHURICHT

Kernexplosionen für friedliche Zwecke

NORBERT SIEBER

HANS-PETER LeIdHOLD

Einführung in die Datenverarbeitung
Festkörperphysik

Entwicklungstendenzen und

Anwendungsmöglichkeiten

Die Schöpfer der physikalischen Optik

Eine Artikelsammlung

HANS-GEORG SCHŌPF

Von Kirchhoff bis Planck

HORST MELCHER

Albert Einstein wider Vorurteile

und Denkgewohnheiten

RENATE WAHSNER

Mensch und Kosmos

Die copernicanische Wende

Helatud Friemel / Josef Brock

Grundlagen der Immunologie

EBERHARD HOEMANN

Funktionelle Blochemie des Menschen

Band 1 und 2

LOTHAR J ÄGER

Grundl ogen

der Klinischen Immunologie

KARLHEINZ LQHS

DIETER MARTINETZ

Entgiftung

Mittei, Methoden und Probleme

JOACHIM NITSCHMANN

Entwicklung bei Mensch und Tier

StePHAN SchNITZLER

Pharmakologische Aspekte

von Immunreaktionen

DIETER SPAAR

HELMUT KLEINHEMPEL

HANS JOACHIM MULLER

Klaus Naumann

Bakteriosen der Kulturpflanzen

Fberhard TEUSCher

Pharmakognosie

Teil I-III 
HEINRIOH BREMER

KLAUS-PETER WendLANDT

Heterogene Katalyse

\section{PETER BIRNER}

HANS-JÖRG HOBMANN

CORNFLIUS WEISS

Mo-theoretische Methoden

in der organischen Chemie

GUNTTHER EPPERT

Einführung

in die Schnelle Flüssigchromatographie

Gerhard Griskler / HeInz SeIdel Die Wasserstoffbrückenbindung

\section{J. G. COLLEE}

Angewandte

medizinische Mikrobiologie

Falizo H. Herrmany

Martina Ch. HerrmanN

Das Hämoglobin des Menschen

Struktur, Funktion, Genetik

Helmot Hrapia

Einführung in die Chromatographie

HANS LUPPA

Grundlagen der Histochemie

Teil I und II

Hasso MEINERT

Fluorchemie

Zur Chemie und Anwendung

Dieter ONKen

Antibiotika

Chemie und Anwendung

BuRkart PhILIPP

GERHARD REINISCH

Grundlagen

der makromolekularen Chemie

HORST REMANE / RAINER HERZSCHUH

Massenspektrometrie

in der organischen Chemie
Vorschau

auf die nächsten Bände:

MaRtin HeINRICh / Hrinz Ulbricht Mechanik der Kontinua

K.́RL LANIUS

Physik der Elementarteilchen

Dieter Mrchel

Grundlagen und Methoden

der kernmagnetischen Resonanz

Gernot NeUgebaUER

Relativistische Thermodynamik

\section{ROBERT ROMPE}

HANS-JUURGEN TREDER

Grundfragen der Physik

Geschichte, Gegenwart und Zukunft der physikalischen Grundlagen-

forschung

JOACHIM SCHUPPAN

Theorie und Methoden

der Konduktometrie

JOACHIM SCHUPPAN

Anwendungen der Konduktometrie

VOLKMAR SCHURICHT

Fusionsreaktoren und Umwelt

KURT SCHWABE

pH-Messung

RAINer SINz

Chronopsychophysiologie,

Chronobiologie und Chronomedizin 


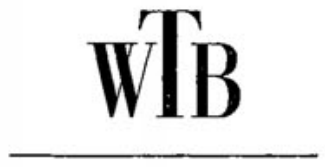

BAND 260

Volker Nollau

\section{Semi-Markovsche Prozesse}

Mit 10 Abbildungen und 5 Tabellen 


\section{Reihe MATHEMATIK UND PHYSIK}

\section{Herausgeber:}

Prof. Dr. phil. habil. W. Holzmüller, Leipzig

Prof. Dr. phil. habil. A. Lösche, Leipzig

Prof. Dr. phil. habil. H. Reichardt, Berlin

Prof. Dr. rer. nat. habil. H.-J. Treder, Potsdam

Verfasser:

Dr. sc. nat. Volker Nollau

Technische Universität Dresden

1980

Erschienen im Akademie-Verlag,

DDR - 1080 Berlin, Leipziger Str. 3-4

Lektor: Dr. Reinhard Höppner

(C) Akademie-Verlag Berlin 1980

Lizenznummer: $202 \cdot 100 / 425 / 80$

Gesamtherstellung: VEB Druckhaus "Maxim Gorki“", 7400 Altenburg

Bestellnummer: 7626131 (7260) · LSV 1074

Printed in GDR

DDR 8,- M 